Anaesthesist 2012 · 61:93-94 DOI 10.1007/s00101-012-2001-9

Online publiziert: 23. Februar 2012

(c) Springer-Verlag 2012

\title{
H. Fink
}

Klinik für Anaesthesiologie, Klinikum rechts der Isar, Technische Universität München

\section{Perioperatives Management und Anästhesie bei Parkinson-Krankheit}

\section{Interdisziplinäre Herausforderung}

Die Parkinson-Krankheit ist, nach der Demenz vom Alzheimer-Typ, die zweithäufigste neurodegenerative Erkrankung. Die altersabhängige Prävalenz in Deutschland beträgt bei Patienten über 65 Jahren 1,8\% [1]. Die Erkrankung verläuft progredient; eine kausale Therapie existiert nicht. Bei einer kleinen Anzahl der Patienten besteht eine genetische Assoziation. Umweltfaktoren, Ernährung und vorangegangene Schädel-Hirn-Traumata (z. B. durch Boxsport) spielen keine oder nur eine geringe Rolle. Interessanterweise scheint Nikotin einen protektiven Einfluss auf die Erkrankung zu haben [2]. Hochrechnungen gehen von einer weltweiten Verdoppelung der Patienten mit Parkinson-Krankheit bis 2030 aus. Obwohl dieser Zuwachs v. a. der steigenden Lebenserwartung in den asiatischen Ländern zugerechnet wird, wird auch in Deutschland die Zahl der erkrankten Patienten in den kommenden Jahren deutlich wachsen [3]. Mit zunehmendem Alter, und damit verbunden, steigender Dauer und Schwere der Erkrankung erhöhen sich auch die Kosten pro Patient deutlich [4]. Komorbiditäten wie Demenz („Parkinson’s disease dementia“) oder Depression sind bei M.Parkinson-Patienten häufiger und verschlechtern die Prognose. Pro Jahr benötigen daher 7-28\% der M.-Parkinson-Patienten einen stationären Krankenhausaufenthalt - ca. 50\% häufiger als die Normalbevölkerung [5]. Dabei ist der Krankenhausaufenthalt eines M.-ParkinsonPatienten länger und häufiger mit Komplikationen behaftet [5].
Für den perioperativ tätigen Anästhesisten stellt das Management elektiver und - noch viel mehr - von notfallmäßigen Eingriffen bei Patienten mit ParkinsonKrankheit daher eine große Herausforderung dar. Anästhesiologisch ist der Patient v. a. durch pharyngoösophageale Dysfunktion [6] mit erhöhter Aspirationsgefahr und kardiovaskuläre Dysregulationen gefährdet, postoperativ durch kognitive Defizite und respiratorische Komplikationen [7]. Ein postoperatives Kältezittern sollte nicht mit M.-Parkinson-typischen Symptomen verwechselt werden. Idealerweise erfolgt die Betreuung der Patienten interdisziplinär; nicht nur von Anästhesist und Operateur, sondern auch zusammen mit einem neurologischen Kollegen. Der von Wüllner et al. verfasste Leitartikel in dieser Ausgabe von Der Anaesthesist nimmt diesen interdisziplinären Gedanken auf.

Grundlage für die optimale perioperative Behandlung eines Patienten mit Parkinson-Krankheit ist die genaue Kenntnis der Pathophysiologie, der Leitsymptome, der Mitbeteiligung anderer Organsysteme sowie der Pharmakodynamik und Interaktion der Pharmaka. Dabei herrscht oft große Unsicherheit bei der Auswahl des Narkoseverfahrens bzw. der zu verwendenden Medikamente, da bei Patienten mit Parkinson-Krankheit Anästhetika im primär und sekundär von der Erkrankung betroffenen Gewebe, also im zentralen und peripheren Nervensystem oder der motorischen Einheit wirken. Um perioperative Symptome nicht fehlzuinterpre- 
tieren, muss streng darauf geachtet werden, die bestehende Basismedikation präund postoperativ weiterzuführen. Ein praktisches Problem ist dabei oft die fehlende Verfügbarkeit der Medikamente auf nichtneurologischen Stationen.

Eine Besonderheit stellt die Anästhesie zur Implantation einer Sonde bei der tiefen Hirnstimulation dar. Zur genauen stereotaktischen Platzierung der Stimulationssonde unter neurophysiologischer und klinischer Erfolgskontrolle werden die meisten Operationen zur tiefen Hirnstimulation beim wachen Patienten durchgeführt. Eine Herausforderung für alle Beteiligten stellt in diesem Fall die lange Operationszeit von mehreren Stunden mit dem Ziel, eine gute Kooperation des Patienten bei vorhandener Symptomatik herzustellen, dar.

Der Leitartikel von Wüllner et al. beleuchtet die verschiedenen Aspekte dieser anästhesiologisch herausfordernden $\mathrm{Pa}$ tienten im Detail, hilft das für den Patienten geeignete Narkoseverfahren zu wählen und schafft somit eine größtmögliche Patientensicherheit.

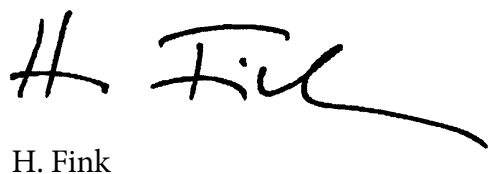

\section{Korrespondenzadresse}

\section{PD Dr. H. Fink}

Klinik für Anaesthesiologie, Klinikum rechts der Isar, Technische Universität München Ismaninger Str. 22, 81675 München h.fink@lrz.tu-muenchen.de

Interessenkonflikt. Der korrespondierende Autor weist auf folgende Beziehungen hin: MSD: Referententätigkeit, Honorare, Reisekostenübernahme; Baxter: Referententätigkeit, Reisekostenübernahme; CareFusion: Honorar, Reisekostenübernahme.

\section{Literatur}

1. Deutsche Gesellschaft für Neurologie (2008) Leitlinie: Parkinson Syndrome, Diagnostik und Therapie. http://www.awmf.org/leitlinien/detail/II/030010.html. Zugegriffen: 08. Januar 2012

2. Ritz B, Ascherio A, Checkoway H et al (2007) Pooled analysis of tobacco use and risk of Parkinson disease. Arch Neurol 64:990-997
3. Dorsey ER, Constantinescu R, Thompson JP et al (2007) Projected number of people with Parkinson disease in the most populous nations, 2005 through 2030. Neurology 68:384-386

4. European Parkinson's Disease Association (o J) The European Parkinson's Disease Standard of Care Consensus Statement. www.epda.eu.com. Zugegriffen: 08. Januar 2012

5. Gerlach OH, Winogrodzka A, Weber WE (2011) Clinical problems in the hospitalized Parkinson's disease patient: systematic review. Mov Disord 26:197-208

6. Troche MS, Huebner I, Rosenbek JC et al (2010) Respiratory-swallowing coordination and swallowing safety in patients with Parkinson's disease. Dysphagia 26:218-224

7. Tzelepis GE, McCool FD, Friedman JH, Hoppin FG Jr (1988) Respiratory muscle dysfunction in Parkinson's disease. Am Rev Respir Dis 138:266-271

\section{J. Benrath, M. Hatzenbühler,} M. Fresenius, M. Heck

\section{Repetitorium Schmerztherapie}

Zur Vorbereitung auf die Prüfung "Spezielle Schmerztherapie"

Heidelberg: Springer 2012, 3., vollständig überarbeitete Auflage, 210 S., (ISBN 978-3642-20023-6), Brosch., 34.00 EUR

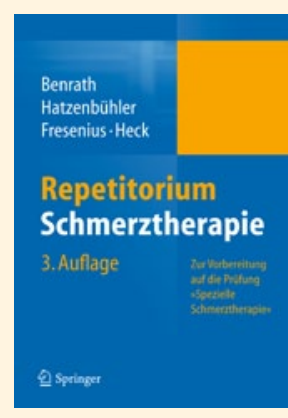

Das Repetitorium Schmerztherapie erscheint nunmehr bereits in dritter, komplett überarbeiteter und aktualisierter Auflage. Den Weiterbildungsinhalten der

Bundesärztekammer für die Zusatzbezeichnung "Spezielle Schmerztherapie" stringent folgend vermittelt es in knapper, aber dennoch verständlicher und einprägsamer Form mit zahlreichen Tabellen und Algorithmen zu Diagnostik und Therapie die wesentlichen Grundlagen über Schmerzentstehung und -verarbeitung, Pharmaund Phytotherapie, invasive Therapieverfahren, Akutschmerztherapie, diagnostische und therapeutische Aspekte der häufigsten Schmerzerkrankungen wie z. B. Tumor-, Kopf- und Rückenschmerzen. Aktuelle Leitlinien zu Rückenschmerzen, dem Fibromyalgiesyndrom und der Langzeitanwendung von Opioiden bei nicht tumorbedingten Schmerzen wurden in diese Neuauflage integriert.

Das Buch ist nicht nur bestens zur Prüfungsvorbereitung zwecks Erlangung der Zusatzbezeichnung „Spezielle Schmerztherapie" geeignet, sondern aufgrund vieler konkreter Praxistipps und nützlicher Hinweise zu Indikationen, Pharmakokinetik und Wirkmechanismen auch ein wertvoller Begleiter im Praxis- und Klinikalltag.

Uwe Junker, Remscheid 